\title{
Minimal spin-one isotriplet dark matter
}

\author{
Alexander Belyaev, ${ }^{1, *}$ Giacomo Cacciapaglia, ${ }^{2, \dagger}$ James McKay, ${ }^{3, ٪}$ Dixon Marin, ${ }^{4, \S}$ and Alfonso R. Zerwekh ${ }^{4, \|}$ \\ ${ }^{1}$ School of Physics \& Astronomy, University of Southampton, Southampton SO17 1BJ, United Kingdom \\ ${ }^{2}$ University of Lyon, Université Lyon 1, CNRS/IN2P3, IPNL, F-69622 Villeurbanne, France \\ ${ }^{3}$ Department of Physics, Imperial College London, Blackett Laboratory, Prince Consort Road, \\ London SW7 2AZ, UK \\ ${ }^{4}$ Departamento de Física and Centro Científico-Tecnológico de Valparaíso, Universidad Técnica \\ Federico Santa María, Casilla 110-V, Valparaíso, Chile
}

(Received 6 November 2018; published 6 June 2019)

\begin{abstract}
In this work we present a simple extension of the Standard Model that contains, as the only new physics component, a massive spin-one matter field in the adjoint representation of $S U(2)_{L}$. In order to be consistent with perturbative unitarity, the vector field must be odd under a $Z_{2}$ symmetry. Radiative corrections make the neutral component of the triplet $\left(V^{0}\right)$ slightly lighter than the charged ones. We show that $V^{0}$ can be the dark matter particle while satisfying all current bounds if it has a mass between 2.8 and 3.8 TeV. We present the current limit on the model parameter space from highly complementary experimental constraints, including dark matter relic density measurement, dark matter direct and indirect detection searches, LHC data on Higgs couplings to photons and LHC data on disappearing track searches. We also show that the two-dimensional parameter space can be fully covered by disappearing track searches at a future $100 \mathrm{TeV}$ hadron collider, which will probe, in particular, the whole mass range relevant for dark matter, thus giving an opportunity to discover or exclude the model.
\end{abstract}

DOI: $10.1103 /$ PhysRevD.99.115003

\section{INTRODUCTION}

Our current microscopic understanding of nature is based on the Standard Model (SM) of particle physics. While the SM successfully describes the properties of all known particles and their interactions, it is widely believed to be incomplete. The shortcomings of the SM are immediately evident when we consider the energy content of the Universe. Cosmological and astrophysical observations show that $26 \%$ of the mass-energy budget for the Universe is composed of an unknown dark matter (DM), while only $5 \%$ is accounted for by ordinary baryonic matter $(5 \%)$. However, despite being a significant fraction of the total mass-energy of the Universe and also having an important role in cosmological structure formation, the nature of DM remains unknown. One of the most popular expectations is that DM could be made up of one or more

\footnotetext{
*a.belyaev@soton.ac.uk

†cacciapa@ipnl.in2p3.fr

\#hmckay93@gmail.com

\$dixonjmp@gmail.com

alfonso.zerwekh@usm.cl
}

Published by the American Physical Society under the terms of the Creative Commons Attribution 4.0 International license. Further distribution of this work must maintain attribution to the author(s) and the published article's title, journal citation, and DOI. Funded by SCOAP. types of weakly interacting massive particles (WIMPs). Many WIMP candidates have been proposed, including scalars [1-4], neutral fermions (the most notable example being neutralinos appearing in supersymmetric models [5]), neutral gauge bosons (such as the Kaluza-Klein excitation of the photon [6-8] in models with universal extra dimensions) and light mesons in composite Higgs models [9-11]. DM particles must be stable on at least cosmological time scales. A popular means of ensuring stability is the introduction of discrete symmetries. For example, the $Z_{2}$ symmetry appears in the form of R-parity in supersymmetry, T-parity in Little Higgs models and KK-parity in extra dimension (see, for example, Refs $[12,13]$ for models with a geometrical origin on the KK parity and Refs [3,14] for minimal cases with an accidental protection).

In this work, we discuss the phenomenology of a novel WIMP candidate. We extend the SM by a massive spin-one matter field in the adjoint representation of $S U(2)_{L}$ (such that the new field is an isotriplet). The main new feature of this model is that no additional states need to be included in the model to comply with gauge invariance and perturbative unitarity of the longitudinal vector polarizations. This allows us to define a truly minimal model, defined in terms of 2 parameters: the mass of DM and its coupling to the Higgs. Furthermore, radiative corrections due to the electroweak gauge bosons ensure that the neutral component is the lightest one. While the case of spin-one DM has 
been studied in the literature before [15-19], these models generally refer to gauge bosons related to new gauge symmetries under which only the dark sector is charged. In our case, the new vector boson is not a gauge boson and is charged under the $\mathrm{SM} S U(2)_{L}$ gauge group. In the absence of electroweak symmetry breaking (EWSB) the requirement of perturbative unitarity automatically imposes a $Z_{2}$ symmetry on this new vector field, thus preventing the lightest (neutral) component from decaying. Therefore this $Z_{2}$ symmetry is a requirement for the consistency of the model rather than simply introduced as a means to stabilize the DM candidate. The presence of the Higgs field and the associated EWSB reintroduces violation of perturbative unitarity. However, this does not break the $Z_{2}$ parity at the level of renormalizable couplings. Furthermore, the loss of perturbative unitarity can occur at a sufficiently high scale, orders of magnitude above the mass of the vector, so that no additional states and/or interactions relevant for the DM phenomenology need to be added to make the model selfconsistent.

The initial motivation for this work, originating from a previous study [20] performed by one of the authors, was the construction of a theory containing a massive matter vector field coupled to a Yang-Mills field (with neither scalars nor symmetry breaking). It has been shown in Ref. [20] that a theory containing a massive vector boson in the adjoint representation, coupled to gauge bosons, is well behaved in the ultraviolet provided that: i) the self-interactions of the new massive vector boson are governed by the same coupling as the gauge interactions, and ii) the new massive vector boson is odd under a $Z_{2}$ symmetry. The latter is a consequence of the absence of trilinear couplings among the new vectors. Additionally, the resulting theory was shown to be Becchi-Rouet-Stora-Tyutin invariant. In some sense, this construction defines a new kind of particle: a vector boson which does not act as the carrier of an interaction but plays the role of dark matter.

The model we will consider has two free parameters, the mass of the vector field and a coupling to the Higgs. The viable parameter space is well constrained by experimental considerations, with an upper bound on the mass coming from a combination of cosmology and DM direct detection experiments. However, the remaining parameter space is difficult to test experimentally. In this work, we evaluate the radiative corrections to the masses of the neutral $\left(V^{0}\right)$ and charged $\left(V^{ \pm}\right)$components of the $S U(2)_{L}$ isotriplet. These radiative corrections generate an essential mass splitting of $\approx 200 \mathrm{MeV}$ which renders the charged state to be short lived, thus making the isotriplet a viable DM candidate. As a result of the small mass difference the dominant decay of the charged component is $V^{ \pm} \rightarrow V^{0}+\pi^{ \pm}$, the width of which we have evaluated using the pion effective theory. It turns out that the $V^{ \pm}$lifetime is on the order of $0.06 \mathrm{~ns}$, thus leading to disappearing charged track signatures from $V^{ \pm}$production, with a characteristic length of $\approx 2 \mathrm{~cm}$ only.
This signature is potentially observable at the LHC and at future high-energy hadron colliders. To find the available parameter space, we have exploited other theoretical and experimental constraints on the model parameter space, such as: i) constraints from perturbative unitarity loss that arise due to the $W^{ \pm}$mass and a nonvanishing coupling of the Higgs field; ii) constraints from Large ElectronPositron (LEP); iii) constraints from the Higgs LHC data; iv) constraints from the DM relic density and v) constraints from DM direct and indirect detection experiments.

The paper is organized as follows: in Sec. II we present the model and compute the radiative correction to the mass of the new particles. In Sec. III we discuss the constraints on the two-dimensional parameter space of the model, thus establishing the up-to-date bounds on the isotriplet mass and identifying the region where the neutral component can account for the DM relic density. In Sec. IV we estimate the reach at the LHC and future hadron collider by means of searches based on disappearing tracks. Finally we present our conclusions in Sec. V.

\section{THE MODEL}

The introduction of massive vector fields into the SM without a companion scalar degree of freedom is generally not desirable. Such theories are not renormalizable and violate perturbative unitarity. However, there are some special cases where these problems can be avoided, such as in the Abelian field theories discussed in Ref. [21]. In Ref. [20] a non-Abelian gauge theory with a massive vector field $(V)$ transforming homogeneously in the adjoint representation of the gauge group was studied. It was found that the theory preserves perturbative unitarity (at least at the tree level) provided that the triple $V$ vertex is absent and that the quartic $V$ coupling is equal but opposite in sign to the quartic gauge boson coupling. The absence of the triple $V$ vertex makes the theory resemble the Abelian case and gives rise to an accidental $Z_{2}$ symmetry. In general, the quartic $V$ vertex would still contribute to unitarity violation for the scattering of the longitudinal component $\left(V_{L} V_{L} \rightarrow V_{L} V_{L}\right)$. However, due to the fact that the quartic $V$ coupling has been linked to the gauge coupling, these dangerous terms are canceled out by a diagram that contains a gauge boson in the t-channel. In this sense, the gauge boson helps unitarizing the $V_{L}$ scattering in the same way as the Higgs boson unitarizes the $W_{L}$ scattering in the SM.

In this work we apply the mechanisms described above to the SM supplemented by a new massive vector boson in the adjoint representation of $S U(2)_{L}$. In other words, we construct an extension of the SM that includes a new massive isotriplet vector boson $\left(V_{\mu}\right)$. By hypothesis, $V_{\mu}$ transforms homogeneously (i.e., $V_{\mu} \rightarrow g_{L}^{\dagger} V_{\mu} g_{L}$ where $\left.g_{L} \in S U(2)_{L}\right)$. Additionally, we impose a $Z_{2}$ symmetry in order to avoid the triple $V$ vertex and we link the quartic 
$V$ coupling to the gauge coupling constant, as found in Ref. [20]. The resulting Lagrangian can be written as:

$$
\begin{aligned}
\mathcal{L}= & \mathcal{L}_{\mathrm{SM}}-\operatorname{Tr}\left\{D_{\mu} V_{\nu} D^{\mu} V^{\nu}\right\}+\operatorname{Tr}\left\{D_{\mu} V_{\nu} D^{\nu} V^{\mu}\right\} \\
& -\frac{g^{2}}{2} \operatorname{Tr}\left\{\left[V_{\mu}, V_{\nu}\right]\left[V^{\mu}, V^{\nu}\right]\right\} \\
& -i g \operatorname{Tr}\left\{W_{\mu \nu}\left[V^{\mu}, V^{\nu}\right]\right\}+\tilde{M}^{2} \operatorname{Tr}\left\{V_{\nu} V^{\nu}\right\} \\
& +a\left(\Phi^{\dagger} \Phi\right) \operatorname{Tr}\left\{V_{\nu} V^{\nu}\right\},
\end{aligned}
$$

where $D_{\mu}=\partial_{\mu}-i g\left[W_{\mu},\right]$ is the usual $S U(2)_{L}$ covariant derivative in the adjoint representation and $\mathcal{L}_{\mathrm{SM}}$ represents the SM Lagrangian. The main difference with respect to the model in Ref. [20] is that the $S U(2)_{L}$ symmetry is broken by the Higgs mechanism and the associated gauge bosons have mass. We thus allow for a coupling of $V$ to the Higgs scalar field $\Phi$. Due to the $Z_{2}$ symmetry the new vector boson does not mix with the gauge bosons when the Higgs field acquires a vacuum expectation value. In this sense, the EWSB is the same as in the SM. Consequently, the physical mass of the new vector bosons, $M_{V}$, is given by

$$
M_{V}^{2}=\tilde{M}^{2}+\frac{1}{2} a v^{2},
$$

where $v \sim 246 \mathrm{GeV}$ is the $\mathrm{SM}$ vacuum expectation value acquired by the Higgs field.

In Secs. III and IV we will consider the phenomenological consequences of varying the two new parameters, $a$ and $M_{V}$. We fix the parameters of the SM to current best-fit values, these are: $\alpha_{\mathrm{EM}}^{-1}=129.950$ for the electromagnetic coupling, $s_{W}^{2}=0.23129$ for the Weinberg angle, $M_{W}=$ $80.385 \mathrm{GeV}$ and $M_{Z}=91.1876 \mathrm{GeV}$ for the electroweak gauge boson masses, and $125.5 \mathrm{GeV}$ for the Higgs mass. In our parameter space scans we consider values of $M_{V}$ from $100 \mathrm{GeV}$ up to $4 \mathrm{TeV}$ and values of $a$ that are within the perturbative regime $(|a| \lesssim 4 \pi)$. This mass range is sufficient to constrain the viable parameter space. To begin with we will focus on the impact of the coupling to the Higgs, $a$, on the perturbative unitarity of the model in the following section.

\section{A. Higgs coupling and perturbative unitarity violation}

Although the model presented in Ref. [20] respects unitary at tree level, our extension of the SM has two sources of unitarity violation. These result from the coupling of the heavy vector to the Higgs field and the fact that the SM $W$ and $Z$ bosons acquire mass via the Higgs itself.

The coupling to the Higgs scalar field, represented by the last term of the Lagrangian in Eq. (1), introduces a new Higgs s-channel contribution to $V_{L} V_{L} \rightarrow V_{L} V_{L}$ scattering. Together with the mass of the $W$ boson, which affects the $t$-channel, it reintroduces perturbative unitarity violation in the model.
In order to estimate the scale of unitarity violation, we study the $V_{L}^{+} V_{L}^{-} \rightarrow V_{L}^{+} V_{L}^{-}$process in the high energy limit. At tree level, seven diagrams contribute to this process in three topologies: the exchange of a Higgs boson, a Z-boson or a photon in the $s$ and the $t$ channels and a contact $V_{L}^{+} V_{L}^{-} V_{L}^{+} V_{L}^{-}$diagram. For large center-of-mass energies the amplitude is

$-i \mathcal{M}=-\frac{\left(16 a^{2} \sin ^{4}\left(\theta_{W}\right)+3 e^{4}\right)(\cos (\theta)+1) M_{W}^{2} s}{8 e^{2} \sin ^{2}\left(\theta_{W}\right) M_{V}^{4}}$.

We then expand the amplitude in partial waves. The most stringent unitarity constraint is obtained for $l=0$, for which the partial amplitude is

$$
a_{0}=\frac{1}{32 \pi} \int_{-1}^{1} \mathcal{M} d \cos (\theta) .
$$

Finally we apply the condition $\left|a_{0}\right|<1 / 2$ which gives the scale of unitarity violation to be

$$
\Lambda \approx \frac{8 \sqrt{\pi} M_{V}^{2}}{\sqrt{4 a^{2} v^{2}+3 g^{2} M_{W}^{2}}} .
$$

As expected this scale goes to infinity when $v \rightarrow 0$ $\left(M_{W} \rightarrow 0\right)$.

In the left panel of Fig. 1 we show the order of magnitude of $\Lambda$ as a function of $a$ and $M_{V}$. We see that for $M_{V} \gtrsim$ $1 \mathrm{TeV}$ (which is, as we will see below, the phenomenologically interesting region) and small $a$ we obtain $\Lambda \gtrsim 100 \mathrm{TeV}$. This indicates that any new sector needed to complete the model and restore unitarity can exist at a high scale. This makes the new sector irrelevant at the Fermi scale, thus rendering our model phenomenologically safe. Small values for the coupling to the Higgs, $a$, imply higher values for the scale of unitarity violation. For increasing $|a|$ the scale $\Lambda$ can become too close to the mass of the vector isotriplet, thus rendering the model inconsistent. The gray area in the plot indicates where $\Lambda<$ $10 M_{V}$ and the theory is thus not reliable.

In principle it would seem convenient to simply fix $a=0$ in order to maximize the scale of unitarity violation, which would be given by

$$
\Lambda(a=0) \approx 150 \mathrm{TeV}\left(\frac{M_{V}}{1 \mathrm{TeV}}\right)^{2} .
$$

However such a choice is not possible since if we eliminate the Higgs- $V$ coupling term at tree level it will be reintroduced by quantum effects at higher loop order. From the Lagrangian in Eq. (1), we can see that loops of the vector isotriplet and the SM $S U(2)_{L}$ gauge bosons contribute to generating an effective coupling between the Higgs field and the triplet. By explicit computation we find that logarithmic divergences arise at one-loop level which require a nonzero counterterm from the $a$ coupling. We can thus estimate the size of $a$ to be: 

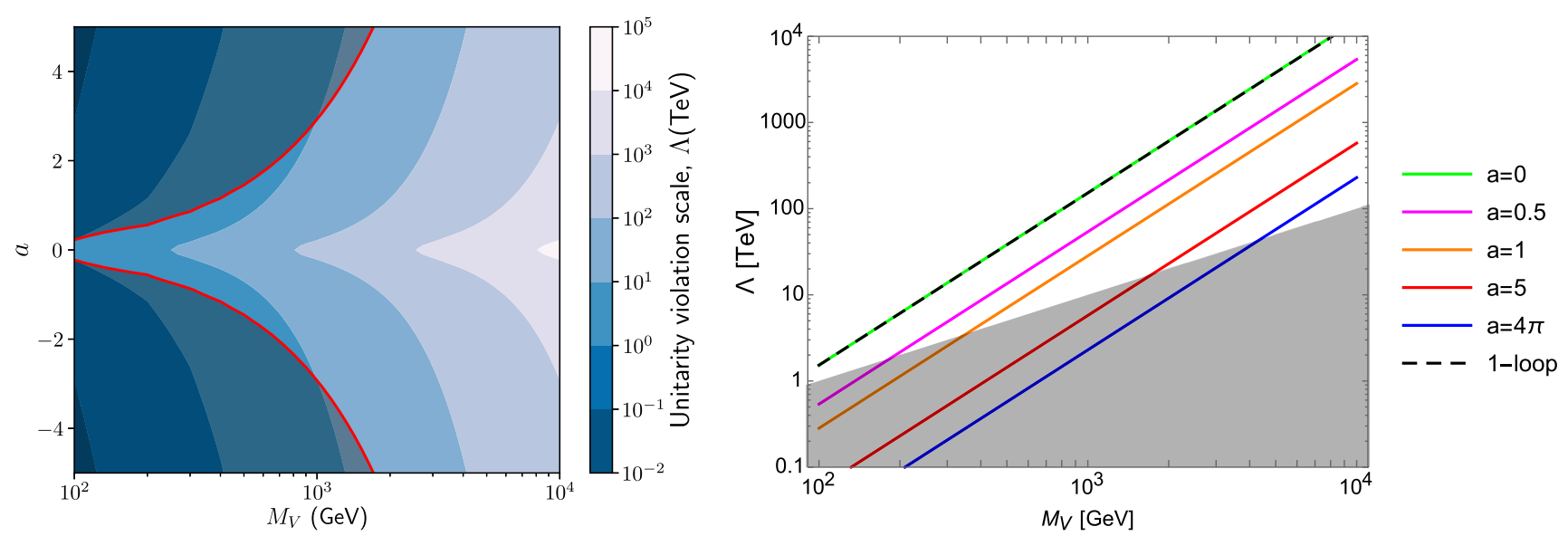

FIG. 1. Left: The expected scale of perturbative unitarity loss as a function of $M_{V}$ and $a$. Areas shaded by colors indicate the scale of unitarity violation $\Lambda$ according to Eq. (5), while area limited by the red contour and shaded by gray color (for both left and right panels) corresponds to a loss of perturbative unitarity at a scale lower than $10 M_{V}$. Right: The expected scale of perturbative unitarity loss for the loop-induced $a$ (dashed), compared to the cases $a=0 ., 1.0,5.0$ and $4 \pi$.

$$
a^{1-\text { loop }}=-3 \frac{\alpha^{2}}{\sin \theta_{W}^{4}} \ln \frac{\Lambda}{M_{V}} \approx-0.0037 \ln \frac{\Lambda}{M_{V}},
$$

where $\Lambda$ is the cutoff of the theory (where we assume $a$ to vanish), and we fix the renormalization scale to $M_{V}$. We identify $\Lambda$ with the scale of perturbative unitarity loss, and plot this using Eq. (5) in the right panel of Fig. 1. This leads to a value of the loop-induced couplings that is mildly dependent on $M_{V}$, with $a^{1-\mathrm{loop}}\left(M_{V}=1 \mathrm{TeV}\right) \approx-0.02$. Figure 1 shows that the impact of the loop induced coupling is always negligible compared to the effect of the $W$ mass, as the dashed line overlaps with the $a=0$ curve. However, as shown in the plot large values of the coupling will significantly lower the expected scale of perturbative unitarity loss. In addition, for fixed $a$ the consistency of the theory requires a lower bound on the mass of the vector field, which ranges between $100 \mathrm{GeV}$ and $1 \mathrm{TeV}$. For masses above $\sim 2 \mathrm{TeV}$, perturbative unitarity violation occurs at sufficiently high scales as long as the coupling $a$ remains perturbative.

The simplest UV completion of the theory would be the introduction of the additional scalar doublet which would generate the mass for the new vector doublet via a spontaneous symmetry breaking mechanism, while the vector doublet would take a role of a gauge field of the symmetry. Eventually this completion predicts the new heavy scalar state with mass between $M_{V}$ and $\Lambda$, the perturbative unitarity violation scale which we have estimated for our minimal model.

\section{B. The radiatively induced mass splitting}

Another important feature of the vector model is the radiatively induced mass splitting between the components. At the tree-level, the neutral and charged isotriplet components are degenerate, having the same mass $M_{V}$.
Fortunately, radiative corrections due to electroweak interactions at the one-loop order and above induce a mass splitting, making the neutral boson lighter than the charged ones. This mechanism is an essential feature of a number of DM theories, including triplet scalar DM [22-25], Minimal DM $[14,26]$ and the wino limit of $R$-parity conserving supersymmetry (where the rest of the supersymmetric spectrum is decoupled and a pure winolike neutralino is a potential DM candidate) [27-30]. This mass splitting is typically found to be on the $\sim 100 \mathrm{MeV}$ scale for DM masses greater than $\sim 100 \mathrm{GeV}$.

We define the physical masses for the charged and neutral components of the vector multiplet as $M_{\text {pole }}^{+}$and $M_{\text {pole }}^{0}$, respectively. These pole masses are given by $p^{2}$ satisfying

$$
p^{2}=M_{V}^{2}-\Sigma_{V}^{i}\left(p^{2}\right),
$$

where $\Sigma_{V}^{i}$ is the real and transverse part of the self-energy for the charged $(i=+)$ or neutral $(i=0)$ component of the multiplet. Equivalently, up to one-loop order, the pole masses are given by

$$
M_{\mathrm{pole}}^{i}=\sqrt{M_{V}^{2}-\Sigma_{V}^{i}\left(M_{V}^{2}\right)} .
$$

The mass splitting between the physical masses of the charged and neutral components can be written by expanding Eq. (9):

$$
\begin{aligned}
M_{\text {pole }}^{i} & =M_{V} \sqrt{1-\frac{\Sigma^{i}\left(M_{V}^{2}\right)}{M_{V}^{2}}} \\
& =M_{V} \sum_{n=0}^{\infty}(-1)^{n}\left(\begin{array}{c}
\frac{1}{2} \\
n
\end{array}\right)\left(\frac{\Sigma^{i}\left(M_{V}^{2}\right)}{M_{V}^{2}}\right)^{n},
\end{aligned}
$$


and taking the difference:

$$
\begin{aligned}
\Delta M & =M_{\text {pole }}^{+}-M_{\text {pole }}^{0} \\
& =M_{V} \sum_{n=1}^{\infty}(-1)^{n}\left(\begin{array}{c}
\frac{1}{2} \\
n
\end{array}\right)\left[\left(\frac{\Sigma^{+}\left(M_{V}^{2}\right)}{M_{V}^{2}}\right)^{n}-\left(\frac{\Sigma^{0}\left(M_{V}^{2}\right)}{M_{V}^{2}}\right)^{n}\right] .
\end{aligned}
$$

For a consistent one-loop result we truncate the expansion to the first term, which appears at order $g^{2}$ in the gauge couplings.

We compute the self-energies in the Feynman-'t Hooft gauge at one-loop order. The one-loop, $\mathcal{O}\left(g^{2}\right)$, mass splitting is obtained from the first term in the expansion in Eq. (11),

$$
\begin{aligned}
\Delta M= & \frac{g^{2}}{12(16 \pi)^{2} M_{V}^{3}}\left[f\left(M_{W}\right)+g\left(M_{W}\right)\right. \\
& -c_{W}^{2}\left(f\left(M_{Z}\right)-g\left(M_{Z}\right)\right) \\
& +5\left(M_{W}^{2}-c_{W}^{2} M_{Z}^{2}\right)\left(A\left(M_{V}\right)-2 M_{V}^{2}\right) \\
& \left.+30 s_{W}^{2} M_{V}^{4} B\left(M_{V}, 0\right)\right],
\end{aligned}
$$

where

$$
\begin{gathered}
f(x)=-\left(30 M_{V}^{4}+26 M_{V}^{2} x^{2}-5 x^{4}\right) B\left(M_{V}, x\right) \\
g(x)=\left(12 M_{V}^{2}-5 x^{2}\right) A(x)
\end{gathered}
$$

and $A$ and $B$ are defined in Eqs. (A1) and (A2), respectively.

To evaluate the mass splitting for $M_{V} \gg M_{W}, M_{Z}$, we use the limits from Eqs. (A3) and (A4), which give

$$
\Delta M=\frac{5 g_{W}^{2}\left(M_{W}-c_{W}^{2} M_{Z}\right)}{32 \pi} \approx 217.3 \mathrm{MeV} .
$$

Like in the fermionic case, this result is independent of $M_{V}$ in the large $M_{V}$ limit. A plot of the full expression in Eq. (12) as a function of $M_{V}$ is presented in Fig. 2 (black solid curve), where we see that the asymptotic constant value is reached for masses above $\sim 500 \mathrm{GeV}$.

The nontruncated mass splitting, Eq. (11), contains higher order terms. The sensitivity of these higher order terms to the renormalization scale can be used to give a naive estimate of the theoretical uncertainty in the one-loop result. The dependence on $Q$ enters both via the input parameters $^{1}$ and via explicit logarithms. In the case of a fermionic multiplet in the large mass limit, only the former

\footnotetext{
${ }^{1}$ For the one-loop mass splitting we need only compute the running $\overline{\mathrm{MS}}$ coupling, which we take as $e_{\mathrm{SM}}\left(m_{Z}\right)=0.3134$, and renormalize using the one-loop $\mathrm{SM}$ renormalization group equation. Any matching to the vector mass is of higher order and thus can be neglected.
}

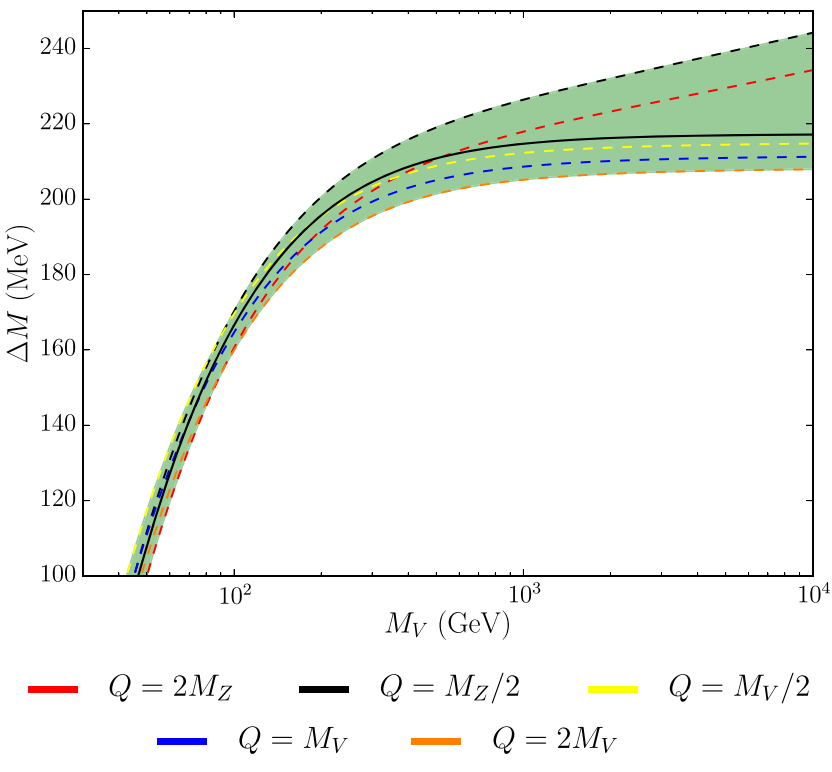

FIG. 2. The one-loop radiatively induced mass splitting between the charged and neutral components of the vector DM. The solid lines represent $\Delta M$ computed at fixed values of the renormalization scale $Q$. The shaded green band indicates the range of values obtained by varying $Q$ continuously between $\min \left\{M_{V} / 2, M_{Z} / 2\right\}$ and $\max \left\{2 M_{V}, 2 M_{Z}\right\}$ and thus constitutes an estimate of the uncertainty on $\Delta M$. The solid black line is the one-loop mass splitting, Eq. (12), with all higher order terms truncated.

$Q$ dependence appears [29,31], thanks to a cancellation of all scale-dependent logarithms between the neutral and charged self-energies: this is due to the fact that the fermionic pole mass is linear in the self-energies, which share the same dependence on the logarithms. The vector case is different because it is the mass squared that depends linearly on the self-energies; thus a cancellation of the explicit logarithms occurs in $\left(M_{\text {pole }}^{+}\right)^{2}-\left(M_{\text {pole }}^{0}\right)^{2}$, but not in $\Delta M=M_{\text {pole }}^{+}-M_{\text {pole }}^{0}$.

In fact, as seen in Eq. (A11), the next-to-leading terms in the series [ $\mathcal{O}\left(g^{n}\right)$ for $\left.n>2\right]$ explicitly contain the term $\log \left(M_{V} / Q\right)$. The dotted lines in Fig. 2 show the results for $Q=M_{Z} / 2,2 M_{Z}, M_{V} / 2, M_{V}$ and $2 M_{V}$. This allows us to identify the theoretical uncertainty with the green region and estimate the error in the range $5-10 \%$. However, this is only a naive estimate for the uncertainty in the one-loop result, and should be considered along with an estimate of the magnitude of missing two-loop corrections, such as that performed for a fermionic multiplet in $[29,30]$.

\section{Electroweak precision tests}

The oblique formalism of Peskin and Takeuchi [32] is a convenient way to parametrize the effect of new physics on electroweak radiative corrections. In an isospin conserving theory, $T$ and $U$ are identically zero; thus we only need compute $S$ which is given by 


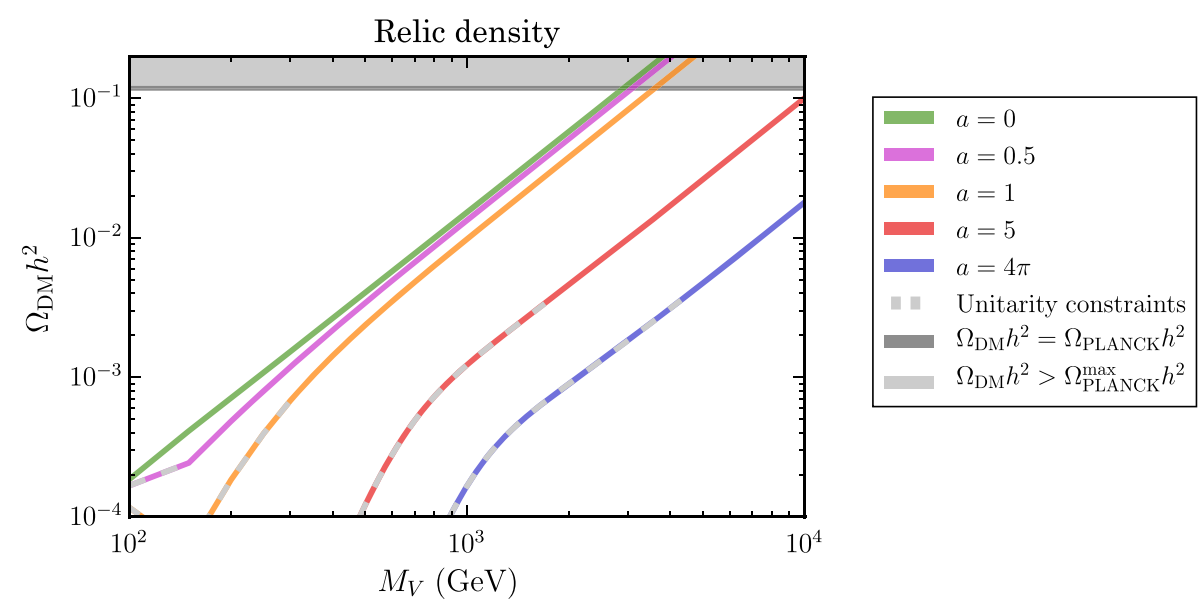

FIG. 3. Thermal relic density for $V^{0}$ vs $M_{V}$ for various values of $a$. Perturbative unitarity loss at $\Lambda<10 M_{V}$ occurs where gray dashing overlays the relic density lines. The gray horizontal band corresponds to the range measured by Planck, $\Omega_{\mathrm{DM}} h^{2}=0.1186 \pm 0.0020$, and the light gray region indicates $\Omega_{\mathrm{DM}} h^{2}>0.1206$.

$S=\frac{4 s_{W}^{2} c_{W}^{2}}{\alpha}\left[\Pi_{Z Z}^{\prime}(0)-\frac{c_{W}^{2}-s_{W}^{2}}{s_{W} c_{W}} \Pi_{Z \gamma}^{\prime}(0)-\Pi_{\gamma \gamma}^{\prime}(0)\right]$

where the $\Pi_{i j}\left(p^{2}\right)$ are the one-loop contributions to the self energies from the new vector field, for the processes $Z \rightarrow Z, \gamma \rightarrow \gamma$ and $\gamma \rightarrow Z$ given by $i j=Z Z, \gamma \gamma$ and $\gamma Z$, respectively. The prime denotes a derivative with respect to the external momentum squared $\left(p^{2}\right)$. With divergences appropriately subtracted, these contributions are found to be

$$
\begin{aligned}
\Pi_{\gamma \gamma}= & \frac{e^{2}}{18\left(16 \pi^{2}\right)^{2}}\left(-2\left(-21 A\left(M_{V}\right)+2 M_{V}^{2}+p^{2}\right)\right. \\
& \left.-3 B\left(M_{V}, M_{V}\right)\left(32 M_{V}^{2}+19 p^{2}\right)\right)
\end{aligned}
$$

and $\tan ^{2} \theta_{W} \Pi_{Z \gamma}=\tan \theta_{W} \Pi_{Z Z}=\Pi_{\gamma \gamma}$. Taking the derivative of each of these and using Eq. (16) we find that $S=0$. Nonvanishing corrections to the oblique parameters will, therefore, only arise at the two-loop level, thus leading to very mild bounds on the mass of the new vector isotriplet.

\section{NUMERICAL RESULTS}

We now turn to analyzing the phenomenology of this vector DM model, with particular attention to the role of the neutral component as a DM candidate.

\section{A. Relic density, direct and indirect searches}

One of the most interesting aspects of this model is the cosmological consequences. For the analysis of these consequences we use the micrOMEGAs package [33]. We start by simply computing the relic density, $\Omega_{\mathrm{DM}}$, of $V^{0}$ for representative values of $M_{V}$ and $a$. Our results are shown in Fig. 3. The dark colored gray horizontal band indicates the region where DM relic density is within one sigma of the value measured by Planck [34], $\Omega_{\text {PLANCK }} h^{2}=0.1186 \pm 0.0020$. The region above this, shaded light gray, indicates where the relic density exceeds the Planck measured value by more than one sigma (and is thus ruled out). The various curves are computed for fixed values of $a$. We see that the DM thermal relic abundance can always match the Planck result for sufficiently large values of the mass $M_{V}$, with the lowest value $M_{V} \approx$ $2.85 \mathrm{TeV}$ attained for $a=0$. For increasing $a$ the mass of the DM increases into the $\mathrm{TeV}$ range, with a maximal value of several tens of $\mathrm{TeV}$ attained at the value of $a=4 \pi$. We also remark that lower values of the mass are also allowed with $V^{0}$ constituting only a fraction of the DM relic density, down to the mass value where perturbative unitarity loss occurs at a too low scale, as indicated by the dashed portion of the curves, where $M_{V}>\Lambda / 10$, with $\Lambda$ given in Eq. (5).

The vector DM can interact with SM nucleons via the Higgs coupling $a$, this allows us to place bounds on the values this coupling may take using limits from direct experiments. There are also loop induced couplings to quarks and leptons, via the electroweak sector, which are sufficiently small to not be of interest in this study. ${ }^{2}$ Finally, a trilinear coupling with the Z-boson $\left(V^{0} V^{0} Z\right)$ is absent in our model. We thus compute the spin-independent scattering cross section on protons, $\sigma_{\mathrm{SI}}$, for various values of $a$, as shown in Fig. 4, and compare them with the 2018 results from the XENON1T experiment $[35,36]$. The region of the parameter space excluded by the perturbative unitarity constraint is indicated by the overlaid gray dashed lines on these lines of fixed $a$. The XENON1T results give significant improvements with respect to LUX [37] and

\footnotetext{
${ }^{2}$ The effective coupling $V^{0} V^{0} \bar{q} q$ is also suppressed by the mass of the quark due to the chirality of the coupling.
} 


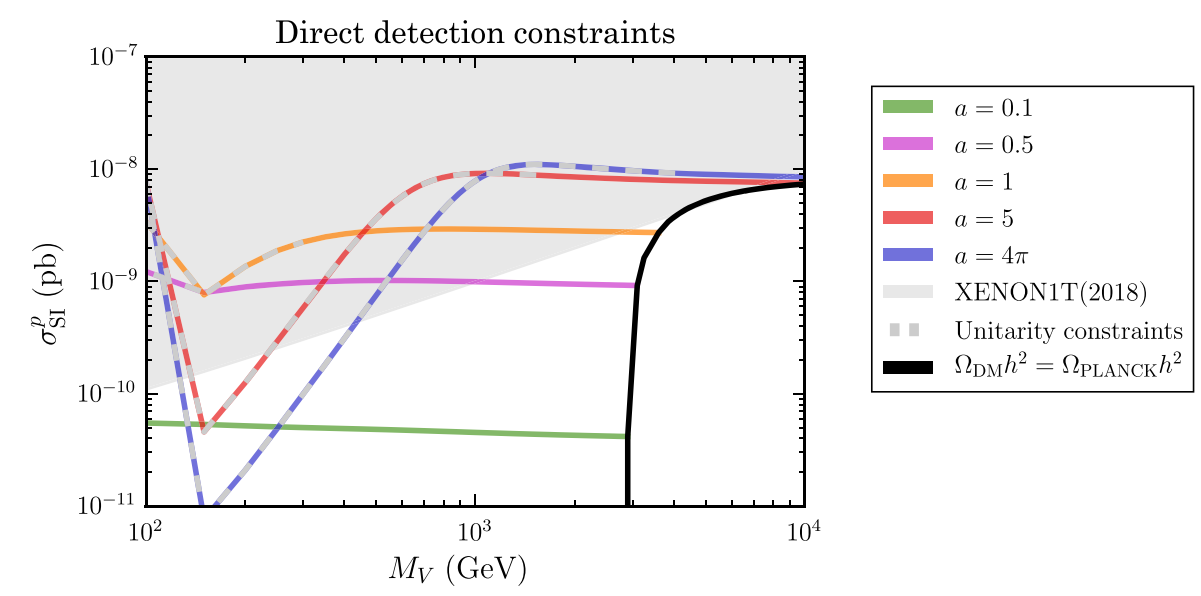

FIG. 4. Spin-independent cross section for $V^{0}$-nucleon elastic scattering as a function of $M_{V}$ and for representative values of $a$. The cross section has been rescaled to take into account the actual thermal relic abundance. The continuous black curve represents the elastic cross section computed with the values of $M_{V}$ and $a$ that saturate the measured DM relic density. The gray dashing highlights the parameter space where perturbative unitarity loss occurs at a too low scale.

PandaX II [38], so we do not consider the latter in this study.

When the thermal relic abundance of vector DM is less than that measured by the Planck experiment, we rescale the cross section appropriately to account for this. The gray line indicates points for which the vector constitutes one hundred percent of the relic abundance of DM. We see that the latest XENON1T results already exclude values of $|a|>1$, even for masses for which the relic abundance is small.

Therefore the DM mass in the surviving parameter space is limited values less than $\sim 4 \mathrm{TeV}$. There is also a surviving region for $|a| \gtrsim 5$ and $M_{V} \gtrsim 8.5 \mathrm{TeV}$. However, due to the large couplings involved in this region our leading order calculations will be unreliable due to large missing higher order corrections, so we currently do not consider this region further. The dip in $\sigma_{\mathrm{SI}}$ at low DM mass $\left(M_{V} \approx M_{h}\right)$ and large $|a|$ is due to resonant Higgs annihilation. However, in this region we find that the scale of perturbative unitarity loss is lower than lower than our constraint $\left(\Lambda<10 M_{V}\right)$. The next update of the XENON detector [36] will improve the constraining power by at least one order of magnitude in the scattering cross section, thus bringing the bound to $|a| \approx 0.3$. Future experiments like LUX-ZEPLIN [39] and PandaX 4T [40] will improve the bound by a further order of magnitude, thus probing down to $|a| \approx 0.1$.

The main annihilation channel for DM in this model is into a $\mathrm{W}$ boson pair, with the final states $Z Z$ and $h h$ also produced via the coupling $a$. Thus the model can be probed by indirect detection experiments looking for cosmic rays generated by these decay products. The most sensitive channel will be into photons emitted by the decay products of the $W^{ \pm}, Z$ and Higgs. As the photon fluxes are very similar for the three final states in our model [41], we compare the velocity averaged cross section of DM with the
HESS results for the $W^{+} W^{-}$channel [42]. The result is shown in Fig. 5, with the cross section rescaled appropriately when the relic abundance is less than that measured by Planck. Note that our annihilation cross section is higher than the usual one considered to saturate the thermal relic abundance because we have coannihilation with the charged vector $V^{ \pm}$. As a result, when saturating the Planck relic density (black line), the lower mass values for $a \simeq 0$, which are not detectable in direct detection experiments, are close to the exclusion deriving from the most optimistic DM profile. Bounds that are less sensitive to the details of the DM profile in the center of the Galaxy can be obtained by observing light emitted by dwarf spheroidal galaxies in experiments like VERITAS [43] and MAGIC [44]. However, these bounds are weaker by at least one order of magnitude. Profile-independent (and thus more robust) bounds can be obtained by detecting distortions of the cosmic microwave background due to photons injected by DM annihilation in the early Universe [45]. However, for heavy DM masses above $1 \mathrm{TeV}$ such bounds are greater than $10^{-24} \mathrm{~cm}^{3} / \mathrm{s}$ and are thus too weak to constrain this model.

\section{B. Contribution to $h \rightarrow \gamma \gamma$}

The charged components of the vector isotriplet contribute to the Higgs boson decay into two photons via loop effects. The Lorentz structure of this contribution is exactly the same as that of the $W^{ \pm}$bosons. This fact simplifies the computation of the partial decay width for this channel. The result, expressed in terms of the partial decay width, is

$$
\begin{aligned}
\Gamma(h \rightarrow \gamma \gamma)= & \frac{\alpha^{2} M_{h}^{3}}{256 \pi^{3} v^{2}}\left[N_{c} Q_{t}^{2} F_{1 / 2}\left(x_{t}\right)+F_{1}\left(x_{W}\right)\right. \\
& \left.+\frac{a}{2}\left(\frac{v}{M_{V}}\right)^{2} F_{1}\left(x_{V}\right)\right]^{2},
\end{aligned}
$$




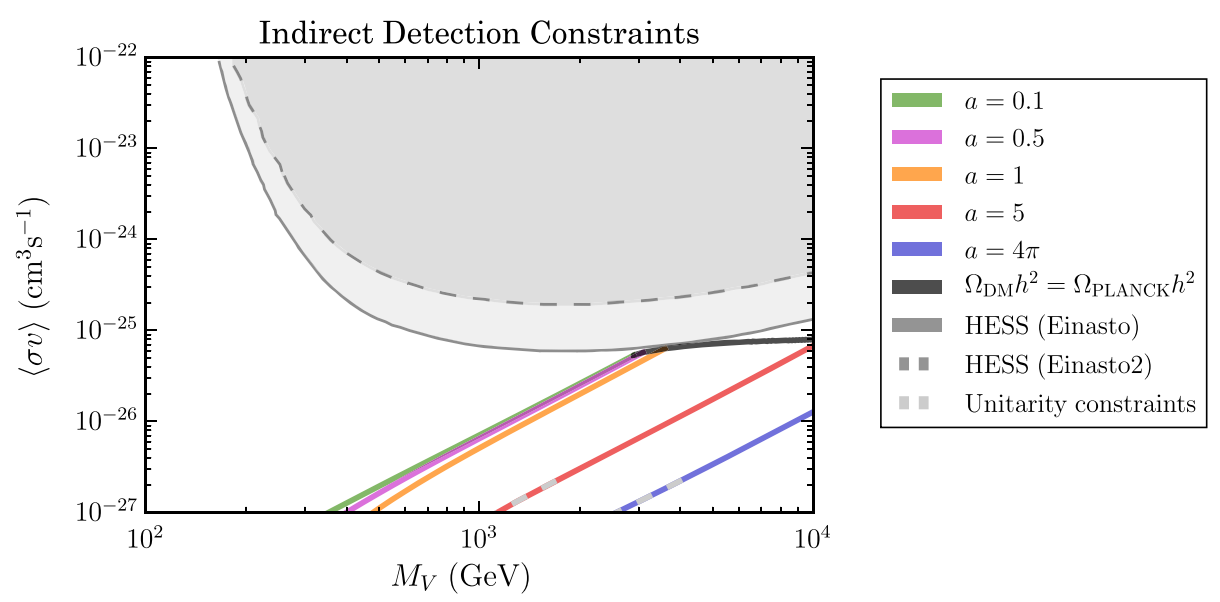

FIG. 5. Annihilation cross section of $V^{0}$ compared to the most recent bounds from HESS in the $W^{+} W^{-}$channel. The two bands consist of two different choices of the DM profile model (Einasto), showing the sensitivity of the bound to the DM distribution at the center of the Galaxy.

where $\alpha$ is the electromagnetic fine-structure constant, $M_{h}$ is the mass of the Higgs boson and $x_{i}=m_{h}^{2} / 4 M_{i}^{2}$. The first term in Eq. (18) represents the contribution of the top quark ( $N_{c}$ and $Q_{t}$ are the number of colors and the electric charge of the top quark, respectively) while the second term originates from the $W^{ \pm}$boson loops. The third term is due to the presence of the $V^{ \pm}$bosons. The functions $F_{1 / 2}$ and $F_{1}$ are the one-loop factors for particles of spin-half and spin-one, respectively, and are defined in Ref. [46]. In this model, only $\Gamma(h \rightarrow \gamma \gamma)$ is sizeably affected by the presence of the vector triplet as other Higgs couplings will only receive next-to-leading order corrections. Therefore we can compare the modification of the partial width, which we embed in the ratio

$$
R_{\gamma \gamma}=\frac{\Gamma(h \rightarrow \gamma \gamma)}{\Gamma(h \rightarrow \gamma \gamma)_{\mathrm{SM}}}
$$

where $\Gamma(h \rightarrow \gamma \gamma)_{\mathrm{SM}}$ is the partial width predicted in the $\mathrm{SM}$, to the signal strength of the diphoton channel measured at the LHC. The most recent results from ATLAS [47] and CMS [48], obtained from an integrated luminosity of approximately $36 \mathrm{fb}^{-1}$, respectively, at $13 \mathrm{TeV}$, give

$$
R_{\gamma \gamma}^{\mathrm{ATLAS}}=0.99 \pm 0.14, \quad R_{\gamma \gamma}^{\mathrm{CMS}}=1.18_{-0.12}^{+0.17} .
$$

The Run-II results significantly improve over Run-I [49] by nearly a factor of two reductions in the error.

To illustrate the numerical impact of the vector isotriplet on the Higgs to diphoton production rates at the LHC, in Fig. 6 we plot $R_{\gamma \gamma}$ as a function of $M_{V}$ for values of $a= \pm 1$ and \pm 5 . The colored bands are the experimentally allowed regions at $95 \%$ confidence level $(2 \sigma)$ from ATLAS (pink) and CMS (yellow). The orange overlapping region is a very conservative estimate of a combination of the two experiments, while a true combination would lead to a significantly larger band [49]. For masses larger than about 1.5 TeV, the computed values of $R_{\gamma \gamma}$ are consistent with reported measurements. We also remark that a cancellation for negative values of $a$ occurs, as seen in the blue lines of Fig. 6. This is due to the cancellation between the top and $W$ loops in the SM, which have opposite signs. A negative $a$ would add to the top contribution, thus eventually overshooting the $W$ loop contribution at large $|a|$ or small masses. In the region relevant for thermal DM, with masses above $2.5 \mathrm{TeV}$, the contribution is smaller than $5 \%$ for $|a|<5$. Therefore it would require a percent level measurement of the diphoton signal strength to be able to probe this model.

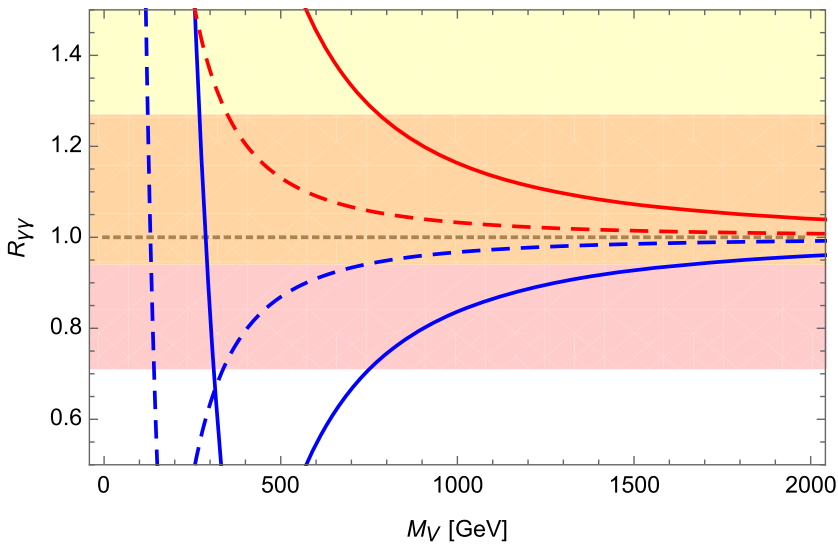

FIG. 6. Contribution of the new vector isotriplet to the $h \rightarrow \gamma \gamma$ decay channel for $a= \pm 1$ (dashed) and $a= \pm 5$ (solid). The color code uses red for positive values and blue for negative. The colored bands are the experimentally allowed regions at 95\% confidence level from ATLAS (pink) and CMS (yellow), while the orange band shows the overlap. 

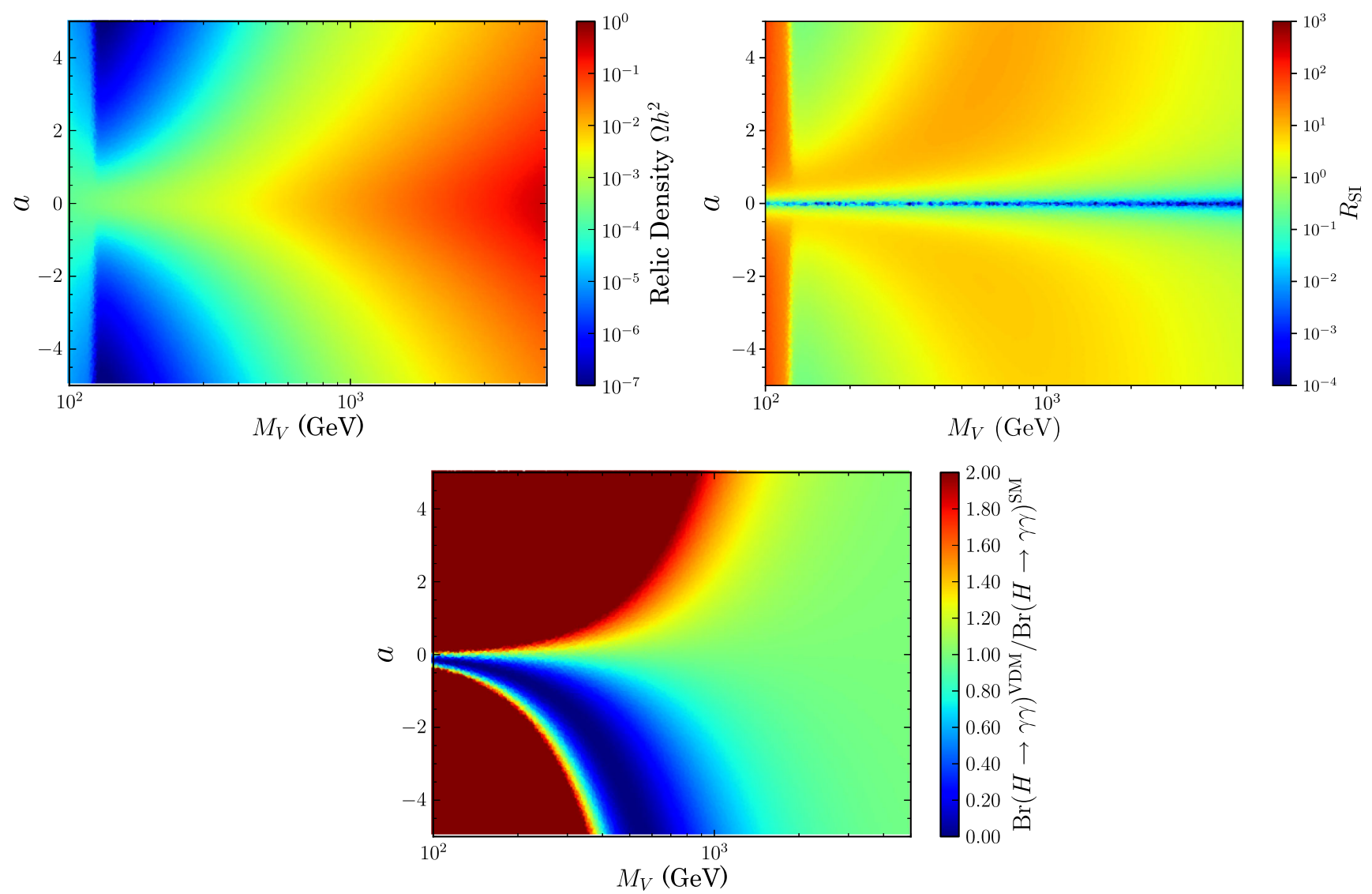

FIG. 7. Contribution of the vector isotriplet to the DM relic abundance (top left), spin-independent direct detection cross section in terms of the ratio in Eq. (21) (top right) and $R_{\gamma \gamma}$ (bottom), as a function of $M_{V}$ and $a$.

\section{Results from the two-dimensional scan}

In Fig. 7 we show the effect of the presence of the vector isotriplet for three observables. In the top left panel we show the DM relic density. In the top right panel we plot the ratio

$$
R_{\mathrm{SI}}=\frac{\sigma_{\mathrm{SI}}^{p}}{\sigma_{\mathrm{SI}, \mathrm{XENON1T}}^{p}} \frac{\Omega_{\mathrm{DM}}}{\Omega_{\mathrm{PLANCK}}},
$$

where $\sigma_{\mathrm{SI}}^{p}$ is the spin-independent scattering cross section of the vector dark matter and $\sigma_{\mathrm{SI}, \mathrm{XENON} 1 \mathrm{~T}}^{p}$ is a parametrization of the central value of the XENON1T limit (given by lower boundary of the gray region in Fig. 4). In the lower panel we present the Higgs partial width into two photons. Note that only the Higgs partial width strongly depends on the sign of $a$. For negative $a$, the isotriplet loop interferes destructively with the $W$ loops, thus reducing the partial decay width of the Higgs in two photons. When the
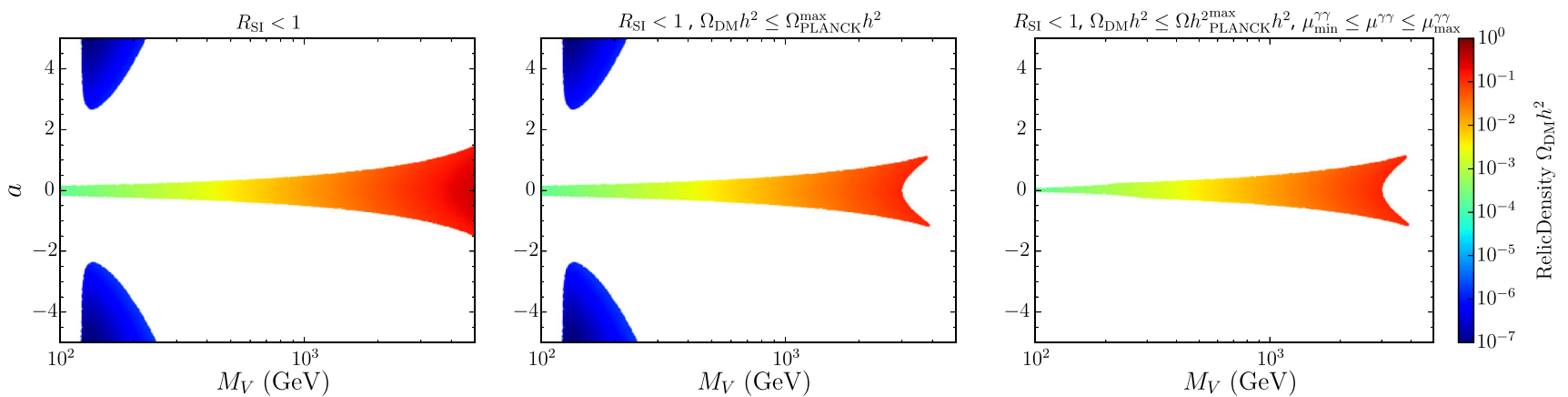

FIG. 8. Impact of experimental bounds on the vector isotriplet parameter space. We progressively impose the constraints from direct detection from XENON 1T 2018 (left), the upper bound on the relic abundance (center), and $R_{\gamma \gamma}$ (right). In Fig. 9 we showed a focused plot on the remaining parameter space with the constraint of the relic abundance within the sigma of the Planck measurement. 
coupling $a$ becomes large, the $V^{+}$loop overwhelms the SM contributions. Thus, there is a thin strip for negative $a$ for which the result accidentally matches the SM result.

Direct bounds on the lifetime of the charged component, $V^{+}$, can be obtained by LEP searches for long lived charginos [50-52] in scenarios with compressed spectra. As the vector has an even larger production cross section than a chargino, we can see that the bound reaches the maximal available mass (half the center-of-mass energy of the collider). Thus, DM masses below $104 \mathrm{GeV}$ are excluded, which is in a range where the perturbative unitarity loss scale is also below our required limit.

In Fig. 8 we show the effect of including constraints from direct detection, consideration of the relic density and measurement of the Higgs coupling to photons. The recent results from XENON1T rule out most of the parameter space, as shown in the left panel, leaving a strip around $a \approx 0$ and two low-relic-density lobes at masses below $1 \mathrm{TeV}$. The upper bound on the thermal relic abundance eliminates the high mass values, as seen in the center panel. In the right panel we impose the $95 \%$ confidence level bound from both ATLAS and CMS on the Higgs to diphoton rate, corresponding to the orange band in Fig. 6. The net effect is that this constraint removes two low mass lobes, while the band around $a \approx 0$ is reduced in width for $M_{V} \lesssim 200 \mathrm{GeV}$. The combination of these constraints provides an upper bound on the Higgs-vector coupling of $|a|<1.2$ at the maximal allowed mass $M_{V} \approx 3.85 \mathrm{TeV}$. Lower masses down to $M_{V}>104 \mathrm{GeV}$ are allowed with smaller values of $a$ and subsequently a lower thermal relic density.

In Fig. 9 we show the remaining parameter space with the additional constraint that the relic abundance of vector DM be within one sigma of the Planck measured value. This requirement imposes a lower bound on the DM mass

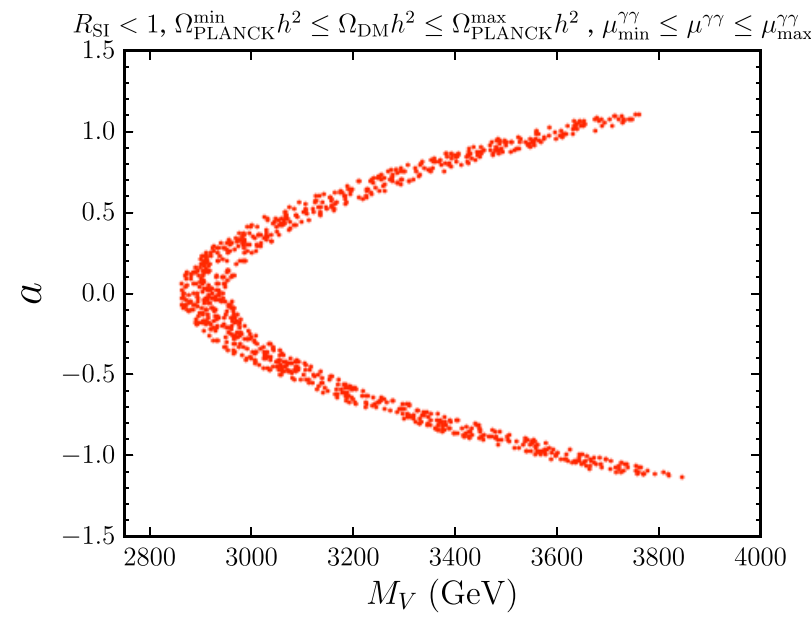

FIG. 9. The remaining parameter space in the vector isotriplet model with constraints imposed from direct detection, the Higgs coupling to photons, and the relic abundance. For this case we demand that the relic abundance of vector DM is within one sigma of the Planck measured value. of $M_{V} \approx 2.85 \mathrm{TeV}$. It is important to note that combined constraints establish upper and lower limits on both $a$ and $M_{V}$, creating the opportunity to completely cover it at future experiments. In Sec. IV we will discuss how the remaining parameter space can be tested using the LHC and future high energy hadron colliders.

\section{PHENOMENOLOGY AT HADRON COLLIDERS}

\section{A. Decay width of $V^{+}$}

Because of the mass splitting computed in Sec. II B the charged component of the vector isotriplet is short lived before decaying into the neutral component plus hadrons or leptons. The main decay channel is the hadronic one, as the kinematically allowed leptonic channels $\left(V^{+} \rightarrow V^{0} e^{+} \nu_{e}\right.$ and $V^{+} \rightarrow V^{0} \mu^{+} \nu_{\mu}$ ) produce partial widths which are at least one order of magnitude smaller. As the small mass gap between $V^{+}$and $V^{0}$ is of the order of the pion mass, the naive perturbative calculation of $V^{+} \rightarrow V^{0} u \bar{d}$ fails to predict the correct width and lifetime of $V^{+}$. For a proper evaluation of the lifetime, which is crucial for collider phenomenology, we have used the effective Lagrangian for $V^{+} \rightarrow V^{0} \pi^{+}$interactions. In momentum space this Lagrangian is

$$
\begin{aligned}
\mathcal{L}_{\pi^{-} V^{+} V^{0}}= & \frac{g^{2} f_{\pi}}{2 \sqrt{2} M_{W}^{2}}\left[\left(p_{V^{+}}-p_{V^{0}}\right)_{\alpha} g_{\beta \gamma}\right. \\
& \left.+p_{V^{0} \beta} g_{\alpha \gamma}-p_{V^{+} \gamma} g_{\alpha \beta}\right] p_{\pi^{-}}^{\alpha} \pi^{-} V^{+\beta} V^{0 \gamma},
\end{aligned}
$$

where $p_{i}$ are the momenta of the respective particles and $f_{\pi}=130 \mathrm{MeV}$ is the usual pion decay constant. This effective Lagrangian is based on the diagram shown in Fig. 10, where the virtual $W$ boson is integrated out and the effective $W-\pi$ mixing is described by the well-known coupling

$$
\mathcal{L}_{W \pi}=\frac{g f_{\pi}}{2 \sqrt{2}} W_{\mu}^{+} \partial^{\mu} \pi^{-}+\text {H.c. }
$$

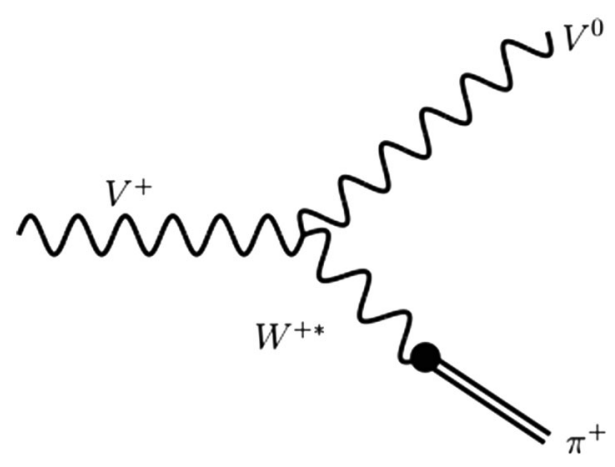

FIG. 10. Feynman diagram of the effective $V^{+} \rightarrow V^{0} \pi^{+}$interaction, obtained by integrating out the off-shell $W$. 

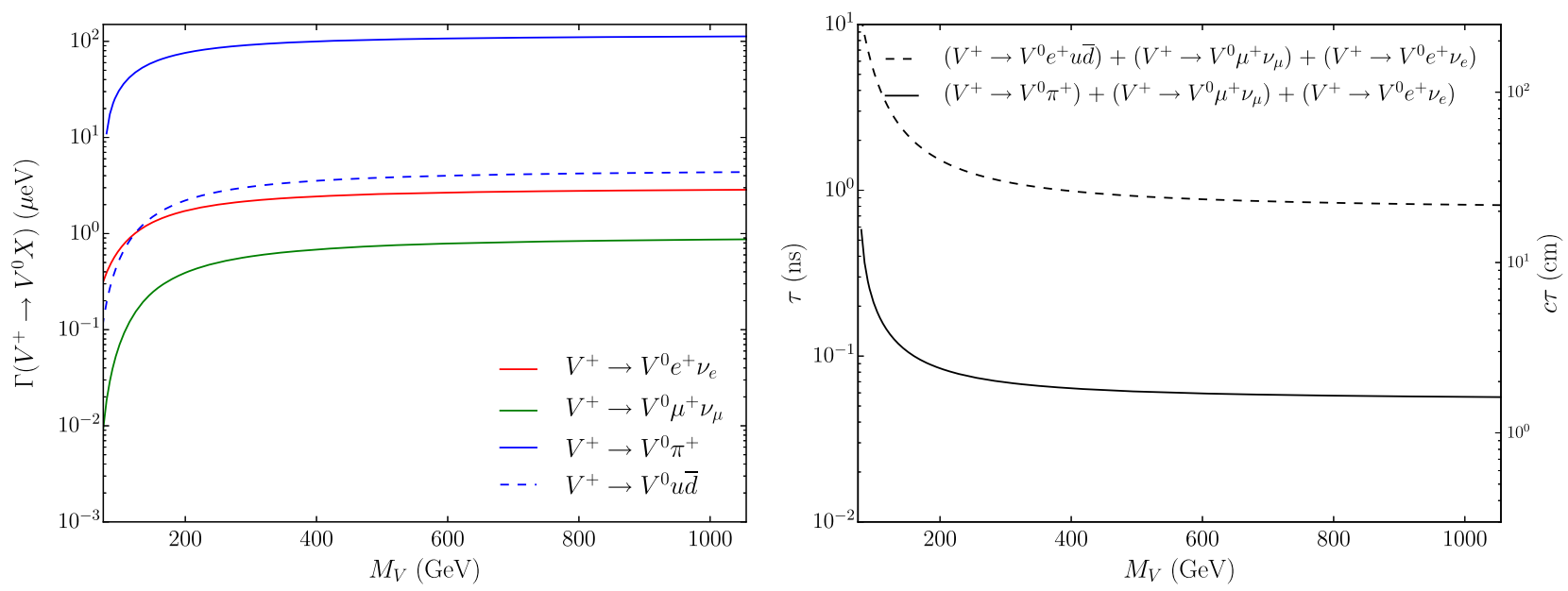

FIG. 11. Left: The partial decay widths of the charged vector isotriplet component in $\mu \mathrm{eV}$ for the three allowed decay channels are given by solid lines. The dashed line indicates the naive perturbative result for the hadronic one. Right: The decay lifetime as a function of the mass, compared to the naive perturbative result.

In the left panel of Fig. 11 we compare the values of the partial decay widths in the various channels, including the naive result for the hadronic one. The decay widths, which are proportional to the mass splitting, tend a mass independent value in the same way as $\Delta M$ does in Fig. 2. We also note that the leptonic channels are subleading compared to the hadronic one, giving $\operatorname{BR}\left(V^{+} \rightarrow V^{0} e^{+} \nu_{e}\right) \approx$ $2.4 \%$ and $\operatorname{BR}\left(V^{+} \rightarrow V^{0} \mu^{+} \nu_{e}\right) \approx 0.65 \%$. Finally we see that the naive calculation for the hadronic channel would predict a partial width roughly one order of magnitude smaller than the one obtained with the effective pion coupling. This results in a $V^{+}$lifetime, shown in the right panel of Fig. 11, of about 0.06 ns, which corresponds to a mean decay length of about $2 \mathrm{~cm}$.

\section{B. Signal at hadronic colliders, LHC and FCC}

Due to the short decay lifetime of the charged isotriplet component most of the $V^{ \pm}$that would be produced at hadronic colliders would decay in a soft pion before hitting the tracker. This would result in missing transverse energy. Therefore typical searches designed for DM should apply, such as monojet searches based on a jet radiated by the initial state. However, the recoil against the jet will boost the produced $V^{ \pm}$, thus allowing a sizeable number of these charged particles to decay inside the tracker. Therefore the most effective way to search for the isotriplet is to look for charged tracks that disappear inside the tracker. This kind of search has been performed by both ATLAS [53] and CMS [54] during Run-I and Run-II, using the electroweak production of charginos in supersymmetry as a benchmark model. In the following, we will reinterpret the ATLAS search for our model, as it is more sensitive to short lifetime charginos. A disappearing track is identified by the inner pixel tracker. An improvement in the ATLAS detector, i.e., the insertion of an inner layer of pixel trackers [55] during the shutdown between Run-I and Run-II, allowed the identification of tracks disappearing between 12 and $30 \mathrm{~cm}$, while the previous Run-I analyses were based on tracks disappearing after $30 \mathrm{~cm}$ [56]. This improvement allows us search particles with shorter decay lifetimes, with the requirement that one jet has a transverse momentum above $140 \mathrm{GeV}$.

We use an implementation of our model in CalcHEP [57], using LanHEP [58-60], to reinterpret these results. The dominant production channel for the vector isotriplet is the same as for winos, supersymmetric partners of W- and Z-bosons, namely electroweak Drell-Yann. As a first test, we computed the jet $p_{T}$ distribution in the two models, as shown in Fig. 12. We see that the $p_{T}$ spectrum is always harder for the vector isotriplet model especially at masses below about $500 \mathrm{GeV}$ for the $\mathrm{LHC}$ at $13 \mathrm{TeV}$. This means that the fraction of $V^{ \pm}$that decays inside the tracker will be

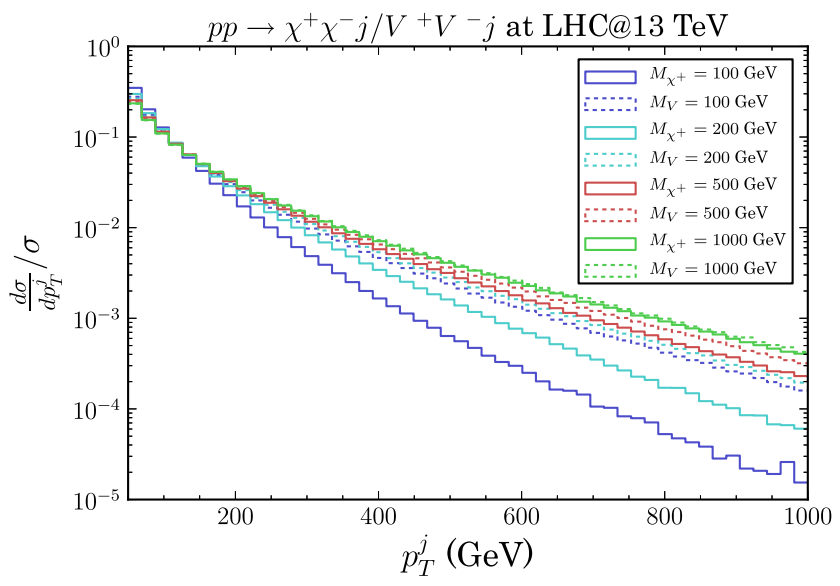

FIG. 12. The shapes of $p_{T}$ distributions for pair production of the charged component of the vector isotriplet and of a wino, for different values of the masses. 


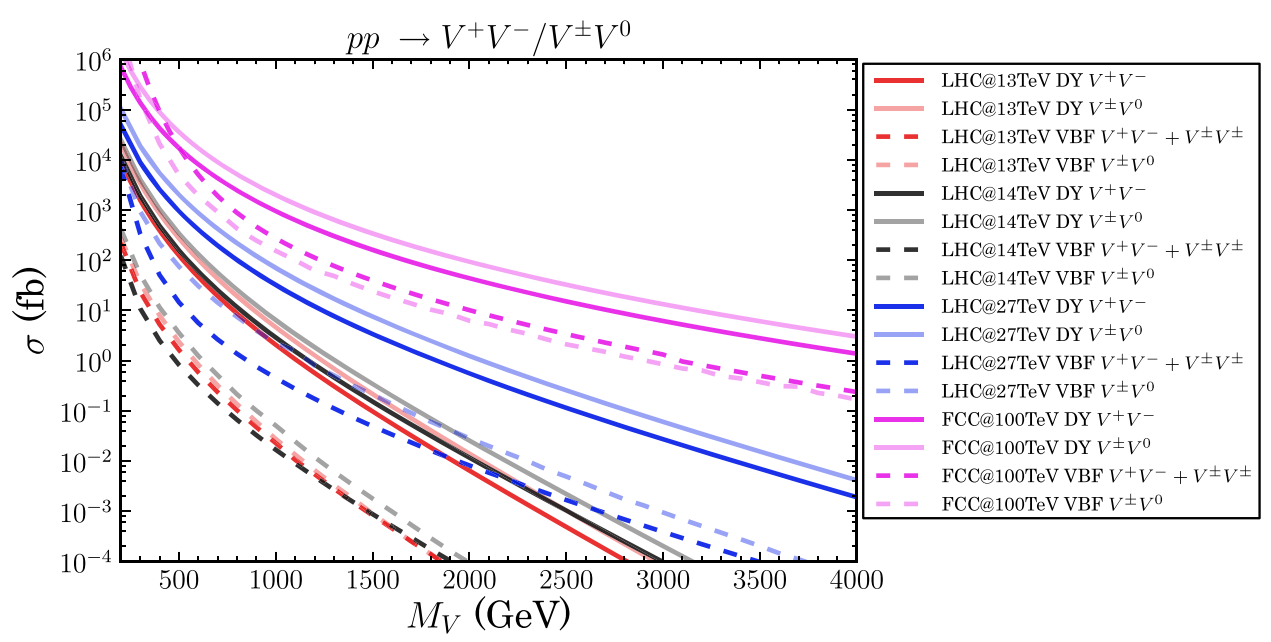

FIG. 13. Production cross sections at leading order for the vector isotriplet model at various LHC energies and for a $100 \mathrm{TeV}$ future collider. The solid lines represent Drell-Yann, while the dashed ones refer to the subleading vector boson fusion channel.

greater for our model than for the ATLAS benchmark one. We thus decided to simply rescale the ATLAS results to the cross sections for the vector isotriplet, knowing that this leads to a conservative estimate of the bound on our model. As ATLAS only provides bounds on the cross section after cuts, we used our simulation to estimate the bound on the pair production cross section for a mean decay length of $2 \mathrm{~cm}$ for $\tau \simeq 0.06 \mathrm{~ns}$.

In Fig. 13 we present production cross sections at leading order for the vector isotriplet model at various LHC energies and for a $100 \mathrm{TeV}$ future collider. The solid lines represent Drell-Yann, while the dashed ones refer to the subleading vector boson fusion channel. In our evaluations we have used NNPDF23 LO as_0130_QED [61], and the QCD scale $Q$ is set to be the invariant mass of the final state

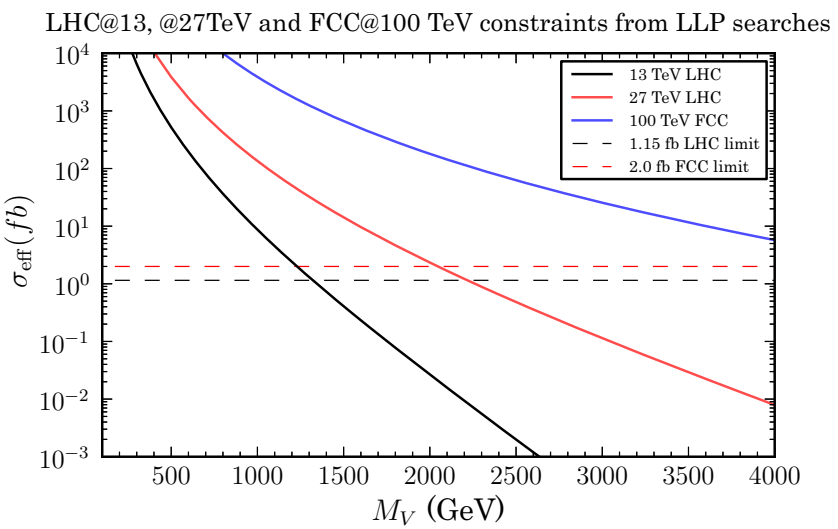

FIG. 14. Effective cross sections $\sigma_{\text {eff }}=\sigma\left(p p \rightarrow V^{ \pm} V^{0}\right)+$ $2 \sigma\left(p p \rightarrow V^{+} V^{-}\right)$at leading order for the vector isotriplet model for 13 and $27 \mathrm{TeV} \mathrm{LHC}$ energies and for a $100 \mathrm{TeV}$ future collider. The dashed black line corresponds the current LHC sensitivity of about $0.85 \mathrm{fb}$ while the dashed red line corresponds to the expected $2 \mathrm{fb}$ sensitivity of $27 \mathrm{TeV}$ LHC and $100 \mathrm{TeV}$ FCC collider in high luminosity regime. particles, $\hat{s}$. We can see that increasing the collider energy has a dramatic effect on the possibility to explore this model, as the cross sections increase significantly for masses in the range of a few TeV. For example, at a future $100 \mathrm{TeV}$ hadron collider the cross section of Drell-Yann production is always above the $1 \mathrm{fb}$ level in the relevant mass range.

To estimate current and future collider sensitivities to the model, we used the following procedure. We have found that for $\tau \simeq 0.06 \mathrm{~ns}$ the current ATLAS limit for the wino mass is about $250 \mathrm{GeV}$ [53], which corresponds to an effective cross section $\sigma_{\text {eff }}=\sigma\left(p p \rightarrow V^{ \pm} V^{0}\right)+2 \sigma(p p \rightarrow$ $V^{+} V^{-}$) of $\sim 1.15 \mathrm{fb}$. By comparing this limit with the respective $\sigma_{\text {eff }}$ from our model, as shown by a black line in Fig. 14, we estimate the current limit on the model to be around $1.3 \mathrm{TeV}$. We remark that this is a rather impressive limit on the mass of a DM candidate when compared to other models. The high-luminosity run of the LHC may improve the bound; however, it will not be able to push it in the interesting range of masses where the vector isotriplet can be the only DM candidate as the cross sections above $2.8 \mathrm{TeV}$ remain too low.

To estimate the reach of a future high-energy collider, we follow the strategy delineated in Ref. [62], where a projection is obtained by increasing the cut on the jet $p_{T}$ in order to match the number of background events of the ATLAS search. ${ }^{3}$ Using the final results of Ref. [62], we estimate that a missing track-based search at a $100 \mathrm{TeV}$ collider will be able to exclude cross sections in the ballpark of $2 \mathrm{fb}$ after an integrated Luminosity of $3000 \mathrm{fb}^{-1}$. From Fig. 14, we see that this would imply a

\footnotetext{
${ }^{3}$ Ref. [62] uses the results from the Run-I analysis [56], but it also implements the Run-II improvement thus assuming reconstructed disappearing tracks at a distance of $10 \mathrm{~cm}$ from the beamline.
} 
reach above $4 \mathrm{TeV}$; thus, probing the entire model will be possible. As a comparison, we also show the cross sections for a high-energy option of the LHC, using the reference value at $27 \mathrm{TeV}$ [63]. Even assuming that the reach in cross section can be as good as for the $100 \mathrm{TeV}$ case, the lower cross sections would only enable us to probe masses up to $\approx 2 \mathrm{TeV}$, still below the interesting range for DM. We conclude that only a $100 \mathrm{TeV}$ future collider would be able to exclude, or discover, this model.

\section{CONCLUSIONS}

In this study we have constructed a minimal extension of the SM by introducing a new massive spin-one isotriplet field. To satisfy perturbative unitarity, this field needs to be odd under a new $Z_{2}$ symmetry, making the neutral component of the triplet a DM candidate. This minimal isotriplet vector DM model introduces only two new parameters: the mass of the DM particle and its coupling to the Higgs boson. We have shown that if the new particle has a mass in the range $2.8 \mathrm{TeV} \leq M_{V} \leq 3.8 \mathrm{TeV}$ the model can explain the measured DM relic density and simultaneously satisfy a number of complementary current experimental constraints. We have also shown that these constraints, ranging from direct DM detection, to LEP bounds and the measurements of the Higgs couplings to photons, also have an important interplay in setting an upper limit on the absolute value of isotriplet coupling to the Higgs (which is currently about one) and the isotriplet mass to be above $100 \mathrm{GeV}$. Masses above $3.8 \mathrm{TeV}$ predict a DM density above the Planck measured value and are thus excluded by the overclosure of the Universe.

The most striking signal at hadron colliders is the presence of disappearing charged tracks coming from the charged component of the vector isotriplet. We calculated the lifetime of this charged component to be $\approx 0.06 \mathrm{~ns}$. A reinterpretation of current ATLAS and CMS searches at the LHC allows us to exclude masses up to $\approx 1.3 \mathrm{TeV}$. It is of worth to note that the exclusion limit we have established is conservative since the transverse momentum distribution of $V^{ \pm}$is harder than that from charginos used in ATLAS analysis, so the respective efficiencies for disappearing charged tracks from our vector DM model are higher, respectively leading to higher signal rate. We have also estimated that future hadron colliders with a center-of-mass energy of $100 \mathrm{TeV}$ would be able to probe masses beyond $4 \mathrm{TeV}$, thus completely covering the allowed parameter space of the model and providing the opportunity to discover or exclude it.

\section{ACKNOWLEDGMENTS}

The authors thank P. Schwaller and J. Zurita for useful discussions and email exchanges on clarifications of the results in Ref. [62]. We would also like to thank A. Pukhov, P. Scott and I. Shapiro for valuable discussions. This work was partially financed by Fondecyt Grants No. 1120346 and No. 1160423, USM internal project USM PIIC program, Conicyt (Chile) Grants No. ACT-1406 and No. PIA/ Basal FB0821. G. C. acknowledges partial support from the Labex-LIO (Lyon Institute of Origins) under Grant No. ANR-10-LABX-66 (Agence Nationale de la Recherche) and FRAMA (FR3127, Fédération de Recherche "André Marie Ampère"). A. Z. is very thankful to the developers of MAXIMA [64] and the package Dirac2 [65]. These software packages were used in parts of this work. A. B. acknowledges partial support from the STFC Grant No. ST/L000296/1, Royal Society Leverhulme Trust Senior Research Fellowship LT140094, and SotonFAPESP grant. A. B. also thanks the NExT Institute and Royal Society International Exchange Grant No. IE150682, partial support from the InvisiblesPlus RISE from the European Union Horizon 2020 research and innovation programme under the Marie SklodowskaCurie Grant Agreement No. 690575. J. M. was funded by the Imperial College London Presidents PhD Scholarship. T. T. and P. M. would like to thank FAPESP for support through Grant No. 2013/01907-0. T. T. would like also to thank FAPESP for support through Grant No. 2016/ 15897-4.

\section{APPENDIX: ONE-LOOP SELF-ENERGIES}

\section{Definitions}

We define the one-loop integrals as

$$
\begin{gathered}
\mathbf{A}(m)=16 \pi^{2} Q^{4-d} \int \frac{d^{d} q}{i(2 \pi)^{d}} \frac{1}{q^{2}+m^{2}} \\
=-\frac{x}{\hat{\epsilon}}+A(x)+\mathcal{O}(\epsilon) \\
\mathbf{B}\left(m_{1}, m_{2}\right)=16 \pi^{2} Q^{4-d} \int \frac{d^{d} q}{i(2 \pi)^{d}} \frac{1}{\left[q^{2}+m_{1}^{2}\right]\left[(q-p)^{2}+m_{2}^{2}\right]} \\
=\frac{1}{\hat{\epsilon}}+B(x, y)+\mathcal{O}(\epsilon),
\end{gathered}
$$

where $d=4-2 \epsilon, 1 / \hat{\epsilon}=1 / \epsilon-\gamma_{\mathrm{E}}+\log (4 \pi) \quad\left(\gamma_{\mathrm{E}}\right.$ is the Euler-Mascheroni constant) and $Q$ is the renormalization scale. For brevity we will write $\mathbf{B}\left(p, m_{1}, m_{2}\right)=\mathbf{B}\left(m_{1}, m_{2}\right)$ when the choice of the external momentum is clear.

We make use of the limiting case when $m_{1}=M \gg m_{2}$ and $p^{2}=M^{2}$ for the integral in Eq. (A2)

$$
\mathbf{B}(M, M, m)=\frac{1}{\hat{\epsilon}}+2-\log \left(\frac{M^{2}}{\mu^{2}}\right)-\frac{\pi m}{M}+\mathcal{O}\left(\frac{m^{2}}{M^{2}}\right) .
$$

The integral in Eq. (A1) is integrated to give 


$$
\mathbf{A}(m)=m^{2}\left(\log \left(\frac{m^{2}}{Q^{2}}\right)-1+\frac{1}{\hat{\epsilon}}\right)
$$

from which the limiting behavior for large $m$ is clear.

\section{Self-energies}

The one-loop self-energies of the charged and neutral components of the vector field $V$ are given by

$$
\begin{aligned}
\Sigma_{V, Z}^{+} & =\frac{19 g^{2}}{6\left(16 \pi^{2}\right)}\left[4-57\left(-c_{W}^{2} \mathbf{B}\left(M_{Z}, M_{V}\right)+\mathbf{B}\left(M_{W}, M_{V}\right)-\mathbf{B}\left(M_{V}, 0\right)\right)\right]+\delta_{Z} \\
\Sigma_{V, M}^{+}= & \frac{g^{2}}{6\left(16 \pi^{2}\right)}\left[1 6 \left(\left(c_{W}^{2} M_{V}^{2}+M_{W}^{2}\right) \mathbf{B}\left(M_{Z}, M_{V}\right)+\left(M_{V}^{2}+M_{W}^{2}\right) \mathbf{B}\left(M_{W}, M_{V}\right)\right.\right. \\
& \left.\left.+M_{V}^{2} s_{W}^{2} \mathbf{B}\left(M_{V}, 0\right)\right)+7 c_{W}^{2} \mathbf{A}\left(M_{Z}\right)+14 \mathbf{A}\left(M_{V}\right)+7 \mathbf{A}\left(M_{W}\right)+20\left(M_{V}^{2}+M_{W}^{2}\right)\right] \\
& \times \frac{a}{16 \pi^{2}}\left(\mathbf{A}\left(M_{H}\right)+2 \mathbf{A}\left(M_{W}\right)+\mathbf{A}\left(M_{Z}\right)\right)-\frac{a^{2} M_{W}^{2}}{\pi^{2}} \mathbf{B}\left(M_{H}, M_{V}\right)+\delta_{M} \\
\Sigma_{V, M}^{0}= & \frac{g^{2}}{3\left(16 \pi^{2}\right)}\left[7 \mathbf{A}\left(M_{V}\right)+7 \mathbf{A}\left(M_{W}\right)+2\left(M_{V}^{2}+M_{W}^{2}\right)\left(5+8 \mathbf{B}\left(M_{W}, M_{V}\right)\right)\right] \\
& \times \frac{a}{16 \pi^{2}}\left(\mathbf{A}\left(M_{H}\right)+2 \mathbf{A}\left(M_{W}\right)+\mathbf{A}\left(M_{Z}\right)\right)-\frac{a^{2} M_{W}^{2}}{\pi^{2}} \mathbf{B}\left(M_{H}, M_{V}\right)+\delta_{M} .
\end{aligned}
$$

We check that the one-loop divergences are canceled by the corresponding counterterms. The required counterterms are

$$
\begin{gathered}
\delta_{Z}=\frac{19 g^{2}}{3\left(16 \pi^{2}\right) \hat{\epsilon}} \\
\delta_{M}=\frac{a^{2} s_{W}^{2}}{\pi^{2} \hat{\epsilon}} M_{W}^{2}+\frac{a}{16 \pi^{2} \hat{\epsilon}}\left(M_{H}^{2}+2 M_{W}^{2}+M_{W}^{2}\right)-\frac{3 g^{2}}{16 \pi^{2} \hat{\epsilon}}\left[M_{V}^{2}+M_{W}^{2}\right] .
\end{gathered}
$$

\section{Series expansion of the mass splitting for large masses}

The one-loop mass splitting is given by the first term of series expansion in Eq. (11). The series expansion can be represented as

$$
\Delta M=5\left(M_{W}-c_{W}^{2} M_{Z}\right) \sum_{n=0}^{\infty} \frac{(-1)^{n^{2}} c_{n}}{16^{n}}\left(\frac{g_{W}^{2(n+1)}}{\pi^{(2 n+1)}}\right)\left(\begin{array}{c}
\frac{1}{2} \\
n
\end{array}\right)\left(42 \log \left(\frac{M^{2}}{Q^{2}}\right)-103\right)^{n}
$$

where we have computed the coefficients $c_{n}$ up to $n=7$ in the limit of large $M_{V}$ and verified that they are convergent.

[1] N. G. Deshpande and E. Ma, Phys. Rev. D 18, 2574 (1978).

[2] L. Lopez Honorez, E. Nezri, J. F. Oliver, and M. H. G. Tytgat, J. Cosmol. Astropart. Phys. 02 (2007) 028.

[3] T. Hambye, F. S. Ling, L. Lopez Honorez, and J. Rocher, J. High Energy Phys. 07 (2009) 090; 05 (2010) 066(E).
[4] A. Belyaev, G. Cacciapaglia, I. P. Ivanov, F. Rojas-Abatte, and M. Thomas, Phys. Rev. D 97, 035011 (2018).

[5] H. Goldberg, Phys. Rev. Lett. 50, 1419 (1983).

[6] K. Griest, Phys. Rev. D 38, 2357 (1988); 39, 3802(E) (1989). 
[7] H.-C. Cheng, J. L. Feng, and K. T. Matchev, Phys. Rev. Lett. 89, 211301 (2002).

[8] G. Servant and T. M. P. Tait, Nucl. Phys. B650, 391 (2003).

[9] M. Frigerio, A. Pomarol, F. Riva, and A. Urbano, J. High Energy Phys. 07 (2012) 015.

[10] Y. Wu, T. Ma, B. Zhang, and G. Cacciapaglia, J. High Energy Phys. 11 (2017) 058.

[11] G. Ballesteros, A. Carmona, and M. Chala, Eur. Phys. J. C 77, 468 (2017).

[12] G. Cacciapaglia, A. Deandrea, and J. Llodra-Perez, J. High Energy Phys. 03 (2010) 083.

[13] G. Cacciapaglia, A. Deandrea, and N. Deutschmann, J. High Energy Phys. 04 (2016) 083.

[14] M. Cirelli, N. Fornengo, and A. Strumia, Nucl. Phys. B753, 178 (2006).

[15] C. Gross, O. Lebedev, and Y. Mambrini, J. High Energy Phys. 08 (2015) 158.

[16] C.-R. Chen, Y.-K. Chu, and H.-C. Tsai, Phys. Lett. B 741, 205 (2015).

[17] A. DiFranzo, P. J. Fox, and T. M. P. Tait, J. High Energy Phys. 04 (2016) 135.

[18] J. Kumar, D. Marfatia, and D. Yaylali, Phys. Rev. D 92, 095027 (2015).

[19] M. Duch, B. Grzadkowski, and M. McGarrie, J. High Energy Phys. 09 (2015) 162.

[20] A. R. Zerwekh, Int. J. Mod. Phys. A 28, 1350054 (2013).

[21] H. Ruegg and M. Ruiz-Altaba, Int. J. Mod. Phys. A 19, 3265 (2004).

[22] P. Fileviez Perez, H. H. Patel, M. Ramsey-Musolf, and K. Wang, Phys. Rev. D 79, 055024 (2009).

[23] O. Fischer and J. J. van der Bij, Mod. Phys. Lett. A 26, 2039 (2011).

[24] T. Araki, C. Q. Geng, and K. I. Nagao, Phys. Rev. D 83, 075014 (2011).

[25] T. Araki, C. Q. Geng, and K. I. Nagao, Int. J. Mod. Phys. D 20, 1433 (2011).

[26] Y. Yamada, Phys. Lett. B 682, 435 (2010).

[27] H.-C. Cheng, B. A. Dobrescu, and K. T. Matchev, Nucl. Phys. B543, 47 (1999).

[28] J. L. Feng, T. Moroi, L. Randall, M. Strassler, and S.-f. Su, Phys. Rev. Lett. 83, 1731 (1999).

[29] M. Ibe, S. Matsumoto, and R. Sato, Phys. Lett. B 721, 252 (2013).

[30] J. McKay and P. Scott, Phys. Rev. D 97, 055049 (2018).

[31] J. McKay, P. Scott, and P. Athron, Eur. Phys. J. Plus 133, 444 (2018).

[32] M.E. Peskin and T. Takeuchi, Phys. Rev. D 46, 381 (1992).

[33] G. Belanger, F. Boudjema, A. Pukhov, and A. Semenov, Comput. Phys. Commun. 185, 960 (2014).

[34] R. Adam et al. (Planck Collaboration), Astron. Astrophys. 594, A1 (2016).

[35] E. Aprile et al. (XENON Collaboration), Phys. Rev. Lett. 119, 181301 (2017).

[36] E. Aprile et al. (XENON Collaboration), Phys. Rev. Lett. 121, 111302 (2018).
[37] D. S. Akerib et al. (LUX Collaboration), Phys. Rev. Lett. 118, 021303 (2017).

[38] X. Cui et al. (PandaX-II Collaboration), Phys. Rev. Lett. 119, 181302 (2017).

[39] D. S. Akerib et al. (LUX-ZEPLIN Collaboration), arXiv: 1802.06039 .

[40] H. Zhang et al. (PandaX Collaboration), Sci. China Phys. Mech. Astron. 62, 31011 (2019).

[41] M. Cirelli, G. Corcella, A. Hektor, G. Hutsi, M. Kadastik, P. Panci, M. Raidal, F. Sala, and A. Strumia, J. Cosmol. Astropart. Phys. 03 (2011) 051; 10 (2012) E01.

[42] H. Abdallah et al. (H.E.S.S. Collaboration), Phys. Rev. Lett. 117, 111301 (2016).

[43] B. Zitzer (VERITAS Collaboration), Proc. Sci. ICRC2015 (2016) 1225.

[44] M. L. Ahnen et al. (Fermi-LAT and MAGIC Collaborations), J. Cosmol. Astropart. Phys. 02 (2016) 039.

[45] T. R. Slatyer, Phys. Rev. D 93, 023527 (2016).

[46] M. Spira, A. Djouadi, D. Graudenz, and P. M. Zerwas, Nucl. Phys. B453, 17 (1995).

[47] M. Aaboud et al. (ATLAS Collaboration), Phys. Rev. D 98, 052005 (2018).

[48] A. M. Sirunyan et al. (CMS Collaboration), J. High Energy Phys. 11 (2018) 185.

[49] G. Aad et al. (ATLAS and CMS Collaborations), J. High Energy Phys. 08 (2016) 045.

[50] A. Heister et al. (ALEPH Collaboration), Phys. Lett. B 533, 223 (2002).

[51] G. Abbiendi et al. (OPAL Collaboration), Eur. Phys. J. C 29, 479 (2003).

[52] J. Abdallah et al. (DELPHI Collaboration), Eur. Phys. J. C 31, 421 (2003).

[53] M. Aaboud et al. (ATLAS Collaboration), J. High Energy Phys. 06 (2018) 022.

[54] A. M. Sirunyan et al. (CMS Collaboration), J. High Energy Phys. 08 (2018) 016.

[55] B. Abbott et al. (ATLAS IBL Collaboration), J. Instrum. 13, T05008 (2018).

[56] G. Aad et al. (ATLAS Collaboration), Phys. Rev. D 88, 112006 (2013).

[57] A. Belyaev, N. D. Christensen, and A. Pukhov, Comput. Phys. Commun. 184, 1729 (2013).

[58] A. V. Semenov, arXiv:hep-ph/9608488.

[59] A. V. Semenov, Nucl. Instrum. Methods Phys. Res., Sect. A 389, 293 (1997).

[60] A. Semenov, Comput. Phys. Commun. 180, 431 (2009).

[61] R. D. Ball, V. Bertone, S. Carrazza, L. Del Debbio, S. Forte, A. Guffanti, N. P. Hartland, and J. Rojo (NNPDF Collaboration), Nucl. Phys. B877, 290 (2013).

[62] R. Mahbubani, P. Schwaller, and J. Zurita, J. High Energy Phys. 06 (2017) 119; 10 (2017) 061(E).

[63] "Hl-lhc Working Group," https://twiki.cern.ch/twiki/bin/ view/LHCPhysics/HLHELHCWorkshop.

[64] Maxima, "Maxima, a computer algebra system. version 5.40.0," (2017).

[65] E. L. Woollett, Dirac2: A high energy physics package for maxima (2012). 